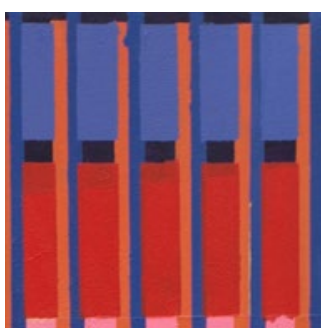

\title{
Conchillas: Un patrimonio nacional único en el Dpto. de Colonia, testimonio de la expansión inglesa de mediados del S. XIX
}

\author{
Conchillas: A Unique National Patrimony in the department \\ of Colonia, as testimony of the English expansion by miD- \\ nineteenth Century
}

\section{Jorge Baeza}

Prof. Adj. Departamento de Arqueología, Facultad de Humanidades y Ciencias de la Educación. Universidad de la República.jorgebaezamartinez@gmail.com

Recibido: 12/07/2017 - Aceptado: 27/08/2017

\section{RESUMEN}

El pueblo de Conchillas tiene su origen en la explotación de áridos, arena y piedra triturada para la construcción del puerto de Buenos Aires, durante la presidencia de Julio Roca, en 1880. La actividad de la Empresa Walker y Co. así como el emprendimiento de Ramos Generales de David Evans, generaron en esta parte del territorio uruguayo un centro urbano, una Factory Town, con características socioculturales y edilicias muy particulares, reconocidas como parte del patrimonio histórico nacional. Ya en el siglo XXI, el proyecto de inversión de la empresa ENCE (productora de pasta de celulosa) que no se concretó, dio lugar a un importante estudio de impacto arqueológico, antropológico e histórico en la zona. Más tarde la empresa Montes del Plata, compró los terrenos de ENCE y desarrolló allí el emprendimiento que había quedado trunco. La empresa, operativa desde mediados de 2014, ha constituido un motor de renovación de actividades y de fuentes laborales para Conchillas y alrededores.

Palabas claves: Conchillas; Factory Town; patrimonio; estudio de impacto; memoria.

\section{ABSTRACT}

The town of Conchillas began with the exploitation of aggregates, sand and crushed stone shipped to build the harbor of Buenos Aires in 1880 when Julio Roca was President. The works 
of Walker \& Co. and the General Store of David Evans generated a Factory Town in this area, an urban development with its own social, cultural and building characteristics that were recognized as national historic monuments. Much later the ENCE entrepreneurship (which didn't finally started to work) gave rise to an important study on archaeological, anthropologic and historic impact on the area. In 2014 the Montes del Plata Company bought the land and started the job intended by ENCE. This turned into a factor to renovate activities in Conchillas and job openings for the region.

Key words: Factory Town; patrimony; impact studies; memory.

"El patrimonio de un pueblo comprende las obras de sus artistas, arquitectos, músicos, escritores y sabios, así como las creaciones anónimas, surgidas del alma popular y el conjunto de valores que dan sentido a la vida, es decir, las obras materiales y no materiales que expresan la creatividad de ese pueblo: la lengua, los ritos, las creencias, los lugares y los monumentos históricos, la literatura y las obras de arte y los archivos y las bibliotecas".'(Conferencia Mundial de Unesco sobre el Patrimonio Cultural”. México D.F. 1982)

\section{Ubicación del Pueblo de Conchillas}

Se sitúa en $\operatorname{los} 34^{\circ} 13^{\prime 2} 2^{\prime \prime}$ de Latitud Sur y 58 03' 03' ' de Longitud Oeste, a unos 50 $\mathrm{km}$ al SO de la Ciudad de Colonia del Sacramento, capital departamental (Figura 1) y a unos $40 \mathrm{~km}$ al NE de la ciudad de Carmelo en el Dpto. de Colonia. Se conecta con la Ruta Nacional 21 a través de un camino vecinal que parte del denominado Puerto Inglés (sobre la costa del Río de la Plata) distante a unos $7 \mathrm{~km}$, atraviesa Conchillas y Pueblo Gil, y finaliza en el punto denominado Radial Fernández, en un recorrido que entre Puerto Conchillas o Inglés y R21, totaliza unos 14 kilómetros (Figura 2).

\section{Su origen}

Dicho núcleo urbano tiene su origen, en la concentración de mano de obra para la explotación minera de áridos (arena y piedra triturada) para la construcción del puerto de Buenos Aires durante la presidencia del Gral. Julio A. Roca, alrededor de 1880 (Figura 3 ). Por este motivo el gobierno uruguayo llamó a licitación de la obra, que fuera obtenida por un consorcio formado por Eduardo Madero e hijos, como concesionarios y por John Hawkshaw, Son \& Haylet como Ingenieros directores y Thomas and Charles Walker\& Co. como Constructores y socios británicos (Borthagaray, 2005). La licitación, en valores de la época, ascendía a unos 7 millones de libras esterlinas, contando con el apoyo financiero de la Casa Baring Brothers de Londres. Las obras comenzaron en 1887 y finalizaron en 1897. Para alcanzar su objetivo la constructora Thomas Walker \& Co. compró tierras al Sr. Luis Gil en el área donde se ubica actualmente la localidad y la zona costera, que lindan al norte con campos del vendedor. Por el Este con el $\mathrm{A}^{\mathrm{o}}$ San Francisco o Conchillas, por el Sur con el Rio de la Plata, y por el Oeste con el Arroyo Limetas. Ocupando una superficie de 625 Há. (Neves, 2007: 8).

El 24 de Octubre de 1887 un representante de la Cía., el Sr. Guilllermo Cotington, llegó a Conchillas junto a sus dos sobrinos de apellido Talbot, con el fin de planificar las actividades de la futura Company Town por lo cual se considera que esa es simbólicamente la fecha fundacional del pueblo. La explotación de las canteras además de Conchillas, generó tres pequeñas poblaciones, Pueblo Nuevo o Punta Pereira, Cantera $N^{o} 5$ y Puerto de Conchillas o Puerto Inglés. Salvo el pueblo de Conchillas y el Puerto, 
los demás han desaparecido. Los trabajos arqueológicos han recuperado recientemente algo de su memoria.

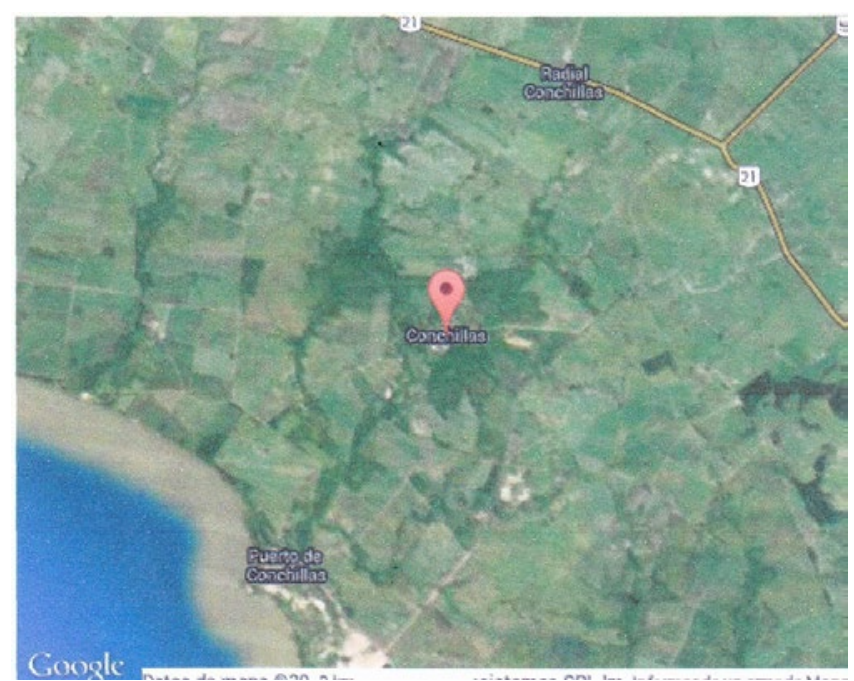

Fig 2. Ubicación de Puerto Inglés, Conchillas-Pueblo Gil y Radial. (Google 2013)

\section{Organización del pueblo y su vida cotidiana}

A partir de la planificación de Mr. Cotington comenzó la construcción de la llamada Factoría; la misma se desarrolló en bloques de viviendas de una planta con paredes gruesas de piedra, techo de zinc a dos aguas sostenido por vigas de madera y cielo raso del mismo material.

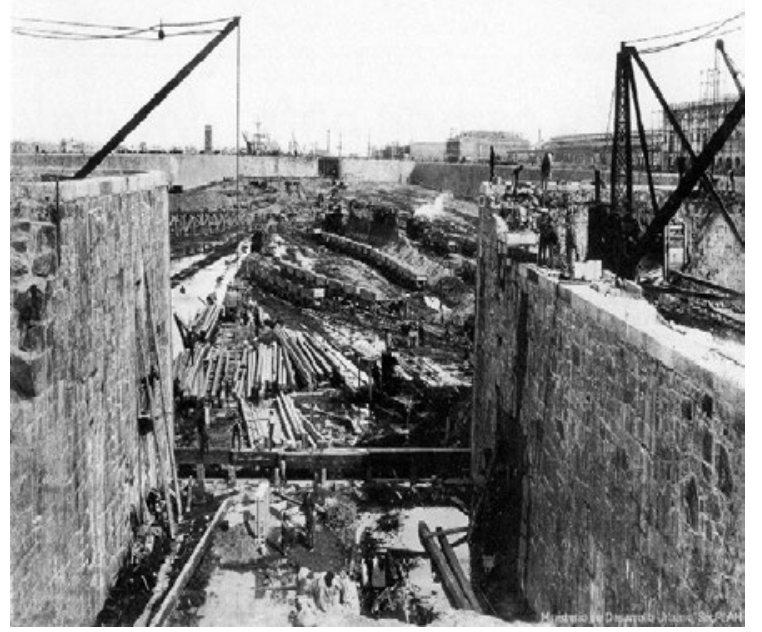

Fig 3. Construcción de Puerto Madero. Foto Pedro Repetto. 
Las viviendas tenían dos habitaciones y cocina. El baño lo constituía una letrina de madera en el patio posterior que disponía de un receptáculo de 20 lts para los desechos orgánicos que un funcionario de la compañía (llamado el Nochero) retiraba todas las noches a través de una pasaje de circulación entre dos bloques de vivienda, en un carro tanque que luego vaciaba en una zona de pozos. Posteriormente aprovechando el sistema ferroviario instalado se utilizaba un vagón tanque y se vertían esos desechos en el Rio de la Plata en un lugar del Puerto Inglés cuyo diseño generaba una corriente que los llevaba rio adentro. Este procedimiento (sin utilizar pozos sépticos individuales) evitó que la napa freática se contaminara. Esto permitió a su vez que el agua distribuida por cañería y surtidores desde un tanque australiano elevado, tomada inicialmente del Rio Conchillas y luego de pozos semi-surgentes con bomba manual, mantuviera su pureza y calidad.

La basura se recogía de lunes a viernes casa por casa y se volcaba en los denominados pozos. Ningún aspecto de la organización de la vida colectiva se dejó al azar para el correcto funcionamiento de la Factoría, en especial la salud del personal y sus familias.

Al personal se le descontaba una pequeña cantidad del salario, por la que tenían derecho, él y su familia, a la asistencia médica del Dr. Kyle, contratado por la Compañía. Esto podría considerarse el primer sistema de tipo mutual en nuestro país. Se construyó además un local como Hospital, en las afueras del pueblo, con el fin de atender los brotes de enfermedades que surgieran, dado el hecho que los obreros venían de Europa y muchas veces eran transmisores de enfermedades del "Viejo Mundo". La Company (como se le llamaba) siguió un patrón de gestión que cubría todos los aspectos; creó también una Escuela para los hijos del personal de la misma, e incluso los menores de la zona circundante. Allí se dictaba clases de $1^{\circ}$ a $6^{\circ}$ año con un docente contratado en Inglaterra por la Compañía; aportando la misma los materiales didácticos. Cada año al finalizar los cursos, cuando los alumnos rendían los exámenes, se terminaba el ciclo con un paseo en Ferrocarril, finalizando con un picnic. Donde se les proporcionaba "limonada y bollos" de la Panadería del Sr. Evans, corriendo los costos por cuenta de la Compañía. Anexo a la Escuela, se edificó un Templo Anglicano donde acudía el personal jerárquico y quienes así lo desearan. Igualmente la empresa creó un área de cementerio, hoy conocido como Cementerio Inglés, donde eran sepultados en un sector exclusivo los de nacionalidad inglesa, y el resto de otras nacionalidades en áreas seleccionadas. Dicho "camposanto" funciona actualmente, ampliado, a cargo de la Junta Local de Conchillas.

Como se puede ver en los registros las nacionalidades eran variadas, de origen europeo mayoritariamente: polacos, checoeslovacos, búlgaros, griegos, húngaros, italianos, españoles, portugueses, todos traídos por la Company en base a campañas promocionales en Europa, de reclutamiento de personal para cubrir los variados oficios necesarios (mecánicos, torneros, matriceros, foguistas, herreros, carpinteros, albañiles, entre otros) para las distintas secciones de la cantera. Arribaban en buques al puerto de Buenos Aires, y desde allí luego eran trasladados a Conchillas.

\section{Infraestructura y organización del trabajo}

La estructura industrial principal estaba vinculada a la cantera, el molino de trituración y al proceso de selección de la piedra, los talleres de atención del equipamiento. Los galpones de estacionamiento de las máquinas estaban estratégicamente en el borde del pueblo y hoy son parte del Patrimonio cultural local. En particular el Taller comprendía 
al aserradero, carpintería, tornería, fundición; todo con la función del mantenimiento del parque industrial. Esta parte era muy importante ya que se desarrollaron dos vías férreas de trocha ancha y de unos $20 \mathrm{kms}$ de extensión, utilizando vías de acero inglés, durmientes de madera dura del Paraguay y también durmientes de hormigón (algo inédito en Uruguay) elementos que aún hoy pueden verse como postes de alambrados que limitan los terrenos y estancias de propietarios de la zona.

Estas vías férreas eran la comunicación con el Puerto Inglés para el transporte de la piedra desde las distintas canteras y posteriormente el transporte de granos desde el comercio del Sr. Evans, socio de la Empresa y abastecedor de mercaderías a la misma, de la población de Conchillas y de toda la zona.

Disponían de cinco locomotoras de 6 ruedas a vapor de alta potencia denominadas "Curva del Llano", "Parish", "Chavarría", "Thorton", y "Gogland". Además había 4 de 4 ruedas de mediana potencia:"Irigoyen", "Ortiz", "Gilmore" y "Zabalía”, (Figuras 4 y 5) que se complementaban con 300 vagones de distinta función para distintas cargas. Las rocas eran extraídas mediante el uso de barrenos de pólvora, de las canteras creadas en bruto con destino a la construcción del puerto de Buenos Aires. Hoy es posible ver todavía estas construcciones y los denominados Polvorines a unos $4 \mathrm{~km}$ del pueblo.

Viejo vagón de la empresa Walker

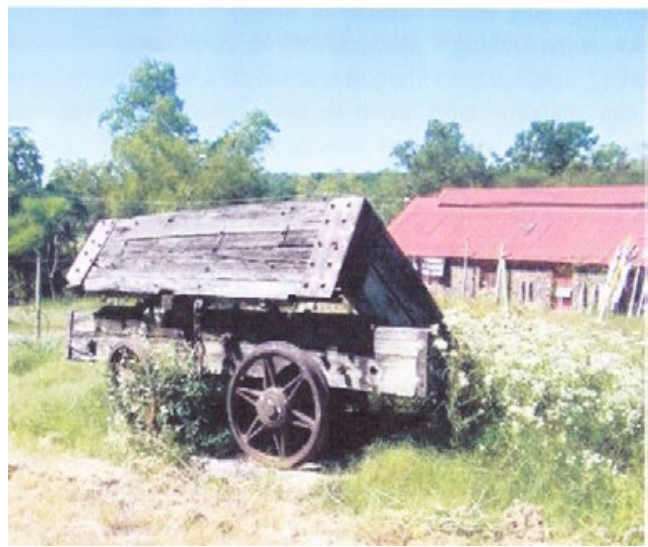

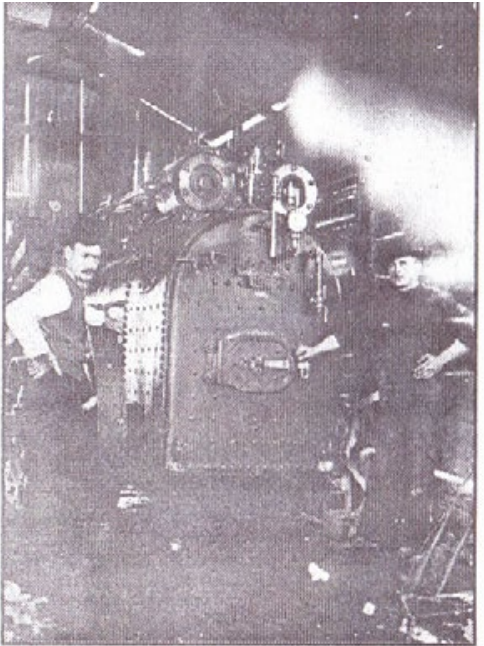

Maquinaria inglesa al servicio de Empresa Walker \& Co.

Fig. 4 y 5. Mostrando maquinaria perteneciente a la Compañía Walker \& Co.

El personal reducía en las canteras con mazas de acero, barretas y marrones de 10 kg., la roca extraída. Luego pasaba al Molino para su trituración y clasificación final. Se almacenaba en tolvas según su granulometría y posteriormente eran cargados en vagones. Estos vagones basculantes (podían volcar el material de costado) se llevaban al puerto donde se descargaban en lanchones rumbo a Buenos Aires.

La arena necesaria para la obra de Puerto Madero (Buenos Aires), dado su proximidad a la costa, se cargaba en vagonetas de "trocha" angosta, de los arenales circundantes y se descargaba en lanchones con un sistema de cinta continua constituida por baldes, cuyo movimiento lo posibilitaban motores eléctricos alimentados por una planta ge- 
neradora en Punta Pereira, donde además existía una planta de fundición para elaborar piezas necesarias para el mantenimiento del área industrial.

Hacia 1922 la empresa Walker electrificó la población con corriente continua, que cobraba según el consumo por familia y KW gastados, a bajo precio.

Con la necesidad de hospedar al personal jerárquico inglés que con motivo del emprendimiento arribaba por distintas tareas, se construyó un Hotel, que disponía de una arquitectura de calidad, con alojamiento para más de 100 personas. Donde todo el equipamiento del hotel había sido traído de Inglaterra contando con personal idóneo para las tareas de atención. Dicho emprendimiento también fue utilizado con fines turísticos, ya que su proximidad con Buenos Aires (35 km por agua) y la existencia de una línea naviera con frecuencias diarias, atrajo a interesados en conocer el lugar, que aseguraban buen clima y descanso, en un espacio de considerable belleza.

En el Puerto Inglés, la Company desarrolló un varadero y taller naval con el fin de reparar sus buques. Como combustible se utilizaba el carbón de hulla, para labores industriales, venido de las minas inglesas en las calderas. A causa de las Guerras Mundiales, se sustituyó el carbón por madera y electricidad. El volumen de trabajadores fue en el momento de máximo crecimiento, del orden de 2500 operarios; la población total de Conchillas alcanzó los 3000 habitantes según el censo de 1908, uno de los primeros a realizarse en Uruguay.

\section{El Almacén de Ramos Generales del Sr. David Evans}

David Evans fue sobreviviente de un naufragio, próximo a la costa de Conchillas. $\mathrm{Su}$ 80 oficio de cocinero facilitó su incorporación a la Walker Co. Con el tiempo, con apoyo de la dirección de la Empresa, Evans, se inició en el Ramo del comercio muy necesario, dadas las dificultades de abastecimiento del sistema. Para ello se construyó con apoyo empresarial un edificio de varias plantas para desarrollar la tarea prevista. El emprendimiento denominado Evans y Cía o Evans Co. en la jerga telegráfica, ya que la empresa poseía Telégrafo para comunicarse con Buenos Aires y el mundo, tuvo un crecimiento notable.

El edificio fue construido alrededor de 1922, en un solo año y con participación de 400 obreros. Allí se podía comprar cualquier artículo de variados rubros, como combustibles (nafta en bidones de 20 lts.) lubricantes, madera, comestibles, automóviles y sus repuestos, productos agrícolas y veterinarios, ferretería y bazar, tienda, sastrería, zapatería, perfumería, cosmética, juguetería y materiales eléctricos.

Este emprendedor inglés vendía al contado, pero normalmente lo hacía con "libreta", cobrando a medida que el cliente podía abonarle, se dice, que sin cobrar intereses.

Fue además prestamista de los agricultores con el fin de facilitar los insumos para la producción. Ello determinó que en su negocio se encontraran desde "clavos, a una cosechadora" y desde "un par de zapatos, a los últimos modelos de automóviles."

El comercio de Evans ofició también de acopiador de granos en sus enormes galpones de chapa que permitían la carga del producto directamente a los vagones con destino a Puerto Inglés y desde allí a Europa. Los envíos se concertaban a través del telégrafo de la Evans Co. La Casa Evans constituyó también un depósito de mercaderías importadas. Su capacidad de emprendimiento lo llevó a crear en una de las habitaciones del enorme edificio Evans, una sala de cine para la población local. Con autorización del Estado y del Banco de la República, acuñó en Amberes (Bélgica) moneda de 5 a 20 centésimos, para las transacciones en la localidad. Se mantuvo soltero, sin descendencia. 
Su contador fue Tomas Kent, un enigmático personaje con una formación y capacidad matemática enorme que llevaba la contabilidad manualmente, de tan formidable comercio. Su origen se presume era ruso blanco, emigrado cuando la revolución bolchevique, esto en base a algunos muy escuetos datos que se conocen de su persona. En la actualidad se conserva el edificio llamado "Casa Evans"; alrededor del 2009 fue embargado por el Banco de la República, rematado y adquirido por la Intendencia de Colonia para ser destinado a actividades culturales. Se conserva como un icono cultural para la localidad de Conchillas.

\section{Casi un final y un renacimiento para el pueblo de Conchillas}

Hacia 1950 el debilitamiento de las actividades a causa de la $2^{\text {a }}$ Guerra Mundial, así como los conflictos diplomáticos entre Uruguay (bajo la presidencia de Luis Batlle) y Argentina (bajo la presidencia de Juan Domingo Perón) hicieron cesar las exportaciones a aquél país, de los materiales de construcción que se producían del lado uruguayo; la industria en Conchillas se vio perjudicada por tal situación. La Empresa Walker y Cia. cerró y vendió sus tierras a la firma Capandeguy y Urrutia, remató todo el equipamiento industrial de la explotación.

Con muy buen criterio urbanístico, dicha firma fraccionó los terrenos del pueblo y la zona rural, y vendió a sus ocupantes los lotes según plano de los Ings. Agrimensores W. y J. Alvariza. Así muchas familias pasaron a ser propietarios de sus casas y solares.

Desde el punto de vista social y especialmente laboral la desaparición de la Walker y Co. constituyó un duro golpe para la localidad de Conchillas hacia la década de 1950.

Siendo una comunidad de tipo "fronteriza" (Romero, 2006) dada su proximidad con Buenos Aires, se originó un "éxodo" del personal técnico y obrero en busca de fuentes laborales, que también se fueron hacia ciudades en el territorio nacional. Si nos remitimos a los censos nacionales vemos la evolución temporal de este fenómeno como indica el cuadro 1.

\begin{tabular}{|c|c|c|c|c|c|c|}
\hline \multicolumn{7}{|c|}{ Evolución demográfica de Conchillas } \\
\hline 1908 & 1963 & 1975 & 1985 & 1996 & 2004 & 2011 \\
\hline 3149 & 825 & 765 & 727 & 784 & 756 & 401 \\
\hline (Fuente: INE $^{2}{ }^{1}$ )
\end{tabular}

Cuadro 1. Evolución demográfica de Conchillas, S XX y XXI.

Dicha tendencia de decadencia del pueblo se revirtió temporalmente en el 2006, cuando se proyectaba la construcción de una Planta de Celulosa por parte de la empresa española ENCE. Así renacieron esperanzas para los pobladores, resignificando los tiempos de bonanza vividos. La "nueva esperanza" empezó para muchos con el regreso a la localidad.

Pero dicho comienzo se vio interrumpido por el retiro de ENCE con motivo de la crisis europea de 2008. Por suerte para los habitantes, la firma Montes del Plata de capitales sueco-finlandeses y chilenos, compró las instalaciones abandonadas por ENCE y renació toda la actividad.

Hacia la mitad de 2014 la planta Montes del Plata quedó operativa y continúa funcionando hasta la actualidad. 
Mucha agua ha pasado bajo los puentes y la localidad dispone finalmente de una nueva fuente laboral, que ha atraído en su pico de construcción a más de 5000 operarios, técnicos de distintas especialidades.

Sólo el tiempo puede confirmar la continuidad del resurgimiento de Conchillas.

\section{Valores patrimoniales}

Por su parte el patrimonio arqueológico, prehistórico, social, histórico y cultural pudo ser salvado, como parte de la identidad de esta tierra coloniense, gracias a un estudio de Impacto realizado por varios equipos dentro de un gran proyecto universitario, que incluyó Arqueología Prehistórica e Industrial, Antropología Social e Historia.

El proyecto de Impacto Arqueológico motivado, entre 2006 y 2008, por la inminente construcción de la Planta de Celulosa y Planta de Energía de Punta Pereira, fue dirigido por el Dr. Antonio Lezama por un Convenio entre la Facultad de Humanidades y Ciencias de la Educación y la Empresa ENCE. Se alcanzaron objetivos previstos en dicho proyecto para la fase de Factibilidad y construcción. Contó con los trabajos pioneros del Liceo de Conchillas (2007), de la obra de Julio Neves (2007), Mario Fripp y otros, asimismo con el aporte de reconocimiento y entrevistas realizadas por un equipo de Antropología Social (dirección de Romero Gorski, S.) permitiendo la comprensión de factores socio culturales presentes en la localidad, considerada una "joya" de urbanización y desarrollo marcado por los valores de la sociedad inglesa victoriana de mediados del siglo XIX e inicios del XX, en un rincón del territorio uruguayo, (Pintos y Lembo, 2009).

Hoy es posible consultar los materiales documentales, los objetos y publicaciones resultantes del trabajo arqueológico, antropológico e histórico y admirar parte de los materiales recuperados, por dicha misión multidisciplinaria, en la Casa de la Cultura de esa localidad.

Así se cumplió una iniciativa que posibilitó mejorar la puesta en valor de esta localidad, con enorme potencial patrimonial y turístico. De hecho el Pueblo de Conchillas y el Hotel Evans, ya habían sido declarados monumentos históricos nacionales (en 1976), pero no se había explorado de forma sistemática y científica sus múltiples facetas.

En el título decíamos "patrimonio nacional único" porque en el territorio uruguayo no existe otro centro urbano, una completa Factory Town de mediados del siglo XIX con las características socioculturales y edilicias de esta localidad.

\section{Bibliografía}

Acevedo,E.(1934). Anales Históricos del Uruguay. Tomo IV. Edit. Casa A.Barreiro y Ramos. Montevideo.

Areco,E. (2005) El impacto de la globalización en la periferia y las nuevas y viejas formas de la dependencia de America Latina.Cuadernos del Cendes.V22 No60.Caracas

Barrán, J. Nahum, B. (1990). ’Batlle, los Estancieros y el Imperio Británico. Tomo I. El Uruguay del novecientos". Ediciones de la Banda Oriental. Montevideo.

Borthagaray,J.M.(2005)'El desarrollo urbano del antiguo Puerto Madero en la ciudad de Buenos Aires". Diseño y Sociedad.

Cabrera, L. Camors,V.(2007) "Los ingleses en nuestro Patrimonio Industrial: el Ferrocarril y el Barrio Obrero de Peñarol”.En:Frega, A. y Vegh, B. (comp).’En Torno a la Invasiones Ingle- 
sas.Relaciones Políticas y Culturales con Gran Bretaña a lo largo de dos Siglos:149-155”. Facultad de Humanidades y Ciencias de la Educación.Udelar. Montevideo.

Campomar,D;Sancassano,L. Szyfer,M.(2006)Las Companys Tows. Como modelo alternativo a la ciudad industrial. Tesis de Grado. ORT.Montevideo.Facultad de Arquitectura .

Conferencia mundial de Unesco sobre el Patrimonio Cultural. (1982) México D.F. 1982.

Cuenca, A. (2006) La colonia británica de Montevideo y la Gran Guerra. Montevideo. Torre del Vigía, Ediciones.

Dupre,H. (1994) Historia del Departamento de Colonia. $2^{\circ}$ Edición.DoloresImpresora Dolores.

Lezama, A. (2008). Estudio de Impacto Arqueológicos y Cultural de la Construcción de la Fábrica de Celulosa y Planta de Energía Eléctrica de Punta Pereira. Convenio DARECOR S.A-UDELAR .Tomo II y III. Informe a la Fac. de Humanidades y Ciencias de la Educación.(inédito). Montevideo.

Liceo de Conchillas. (2007). Resignificación de la Memoria Comunitaria en pos de la proyectiva social merecida.Colonia.Imprenta Logos.

Nemmer, A. (2003) Apuntes para una historia de Conchillas.C.H.Walker y Cía Ltda 1887-1950. David Evans , 1910. Tomas Kent. pp 1-14.Montevideo.

Neves, J. (2007) Canteras y Médanos.Montevideo, Edit Gráfica Umpierrez. S.A.

Pintos,M. y Lembo,V. (2009) El rol de la Antropología Social en el Estudio de Impacto Arqueológicos y Cultural, 163-178. En: Anuario de Antropología Social y Cultural en Uruguay. Romero, S. (Editora) Montevideo, Nordan-Comunidad.

Romero,S. (2001a) Estudio sobre la situación fronteriza en Colonia del Sacramento. En:Anuario de Antropología Social y Cultural. Romero, S. Montevideo, Nordan- Comunidad.

Romero, S. (2001b) Uruguay dentro del continuum urbano de la sub-región Plata-MERCOSUR (CLACSO-ASDI). En: The European Journal of Planning. www.planum.net

Varese, J. (compilador) (2010). Influencia Británica en el Uruguay. Aportes para su historia. Montevideo. Ediciones Cruz del Sur. 
